\title{
Clinical significance and expression of microRNA in diabetic patients with erectile dysfunction
}

\author{
XI JIANG $^{1,2^{*}}$, YONG LUO $^{3 *}$, SHULI ZHAO $^{4}$, QICHAO CHEN $^{1}$, CHAO JIANG $^{1}$, \\ YUTIAN DAI ${ }^{5}$, YUN CHEN ${ }^{5}$ and ZHIGANG CAO ${ }^{1}$ \\ ${ }^{1}$ Department of Urology, Nanjing First Hospital, Nanjing Medical University, Nanjing, Jiangsu 210006; \\ ${ }^{2}$ Department of Urology, Huai'an First People's Hospital, Nanjing Medical University, Huai'an, Jiangsu 223300; \\ ${ }^{3}$ Department of Endocrinology and ${ }^{4}$ Center Laboratory, Nanjing First Hospital, Nanjing Medical University, \\ Nanjing, Jiangsu 210006; ${ }^{5}$ Department of Urology, Nanjing Drum Tower Hospital, \\ Nanjing University Medical School, Nanjing, Jiangsu 210008, P.R. China
}

Received August 28, 2014; Accepted February 24, 2015

DOI: 10.3892/etm.2015.2443

\begin{abstract}
The aim of the present study was to investigate the expression of microRNA (miR)-93, miR-320 and miR-16 and to assess their diagnostic value in diabetic patients with erectile dysfunction (ED). A total of 120 individuals were divided into three groups, which included the diabetics with ED group (ED group), the diabetics without ED group (NED group) and the healthy volunteers group (control group). Each group included 40 individuals. Serum samples were collected and reverse transcription quantitative polymerase chain reaction detection of the three types of miRNA was performed and the sensitivity of ED was analyzed by receiver operating characteristic curves. A negative correlation was identified between the incidence of ED in patients with diabetes and serum total testosterone levels $(\mathrm{r}=0.302, \mathrm{P}<0.05)$; however, a positive correlation was observed between the incidence of ED in diabetics and the HbA1c level $(r=0.231, \mathrm{P}<0.05)$. Additionally, the relative expression levels of the three types of miRNA were higher in the ED group when compared with the NED and control groups $(\mathrm{P}<0.05)$. When compared with the control group, the area under the curve (AUC) values for miR-93, miR-320 and miR-16 were $0.793,0.818$ and 0.810 , respectively, in the ED group and $0.576,0.532$ and 0.542 in the NED group, respectively. Furthermore, when compared with the NED group, the AUC value for miR-93, miR-320 and miR-16 was $0.707,0.810$ and 0.833 , respectively, in the ED group. Therefore, the expression levels of miR-93, miR-320
\end{abstract}

Correspondence to: Dr Zhigang Cao, Department of Urology, Nanjing First Hospital, Nanjing Medical University, No. 68 Changle Road, Nanjing, Jiangsu 210006, P.R. China

E-mail: zhigangcaocn@126.com

*Contributed equally

Key words: diabetes, erectile dysfunction, microRNA, serum, testosterone, glycated hemoglobin and miR-16 may be useful for the early diagnosis of ED in patients with diabetes.

\section{Introduction}

Diabetes comprises a group of metabolic diseases in which blood sugar levels are high for a prolonged period of time. A Chinese epidemiological survey in 2010 revealed that the total prevalence rate of diabetes was up by $9.7 \%$ (1). Erectile dysfunction (ED) is sexual dysfunction characterized by the inability to develop or maintain an erection of the penis during sexual activity over the course of three months (2), and is one of the chronic complications in males with type 2 diabetes. Numerous studies have reported that the incidence of ED in diabetic individuals varies between 30 and $80 \%$, which is six times higher compared with normal individuals, and augments with an increase in age and duration of diabetes $(3,4)$. The pathogenesis of diabetic ED is complex. Diabetic angiopathies include macrovascular disease, microvascular disease and endothelial dysfunction, which are all known to be involved in the pathophysiological process of ED (5-7).

Generally, a diagnosis of ED depends on the illness history, the international index of erectile function-5 (IIEF-5) and the hardness scale of penile erection $(8,9)$. However, ED is often a private matter and considering that patients are not recognizing ED, there is a doubt that the aforementioned tests will diagnose ED. Evidently, there are other methods for diagnosing ED, such as nocturnal penile tumescence, magnetic resonance angiography, corpus cavernosometry and penile nerve function. However, these methods are complex and traumatic; thus, have not been adapted to clinical application (10).

microRNA (miRNA) are small non-coding RNA molecules (containing 22 nucleotides) found in animals and plants, which functions in transcription and post-transcription to negatively regulate gene expression (11). Certain miRNA molecules are used as biomarkers since they are present in the serum. Furthermore, previous studies have indicated that miRNA plays an important role in the development of diabetes and diabetic complications (12-15). The current preliminary trial investigated the relevance of miR-93, miR-320 and miR-16 in 
the incidence of diabetic ED. Reverse transcription quantitative polymerase chain reaction (PCR) was used to detect the expression levels of the miRNAs in diabetic patients with ED, and the clinical value of the miRNAs was investigated as diagnostic evidence for diabetic ED.

\section{Materials and methods}

Subjects. In total, 40 diabetic patients with ED (ED group) were recruited from Nanjing Hospital of Nanjing Medical University (Nanjing, China) between July 2012 and December 2013. The mean age was $47.37 \pm 5.81$ years, and mean diabetes duration was $5.40 \pm 2.56$ years. The diagnosis criteria of diabetes was in accordance with the World Health Organization's criteria (16), and IIEF-5 scores were used to assess whether the diabetic patients suffered from ED. In addition, 40 diabetic patients without ED (NED group) were selected. The mean age was $46.05 \pm 4.88$ years, and the duration of diabetes was $5.72 \pm 2.33$ years. In addition, 40 healthy individuals from the same community, who did not have any organic lesions, were selected as a control group; the mean age was $43.9 \pm 5.69$ years. No statistically significant differences were observed among the three groups with regard to age or the duration of diabetes (between the ED and NED groups). The study was conducted in accordance with the Declaration of Helsinki and with approval from the Ethics Committee of Nanjing Medical University. Written informed consent was obtained from all the participants.

Clinical data. IIEF-5 scores were obtained using a questionnaire. Body mass index (BMI) was calculated by formula: $\mathrm{BMI}=$ Body weight $/$ height $^{2}\left(\mathrm{~kg} / \mathrm{m}^{2}\right)$. Levels of fasting plasma glucose (FPG), glycated hemoglobin (HbAlc), total cholesterol (TC), triglyceride (TG) and total testosterone (TT) were determined using an exsanguinate assay. The presence of macroangiopathy (MA), diabetic retinopathy (DR) and diabetic nephropathy (DN) were evaluated by vascular B ultrasound, microalbuminuria test and fundus examination.

Blood sample collection. Peripheral blood samples (3 ml) were collected in the morning and stored in coagulation-promoting tubes (Nanjing KeyGen Biotech Co., Ltd., Nanjing, China). Samples were then centrifuged at 2,054 $\mathrm{x} \mathrm{g}$ for $5 \mathrm{~min}$ and the supernatant was stored at $-80^{\circ} \mathrm{C}$ in the centrifuge tube.

Total RNA extraction. A 100- $\mu$ l sample of serum was treated with phenol and chloroform (Sigma-Aldrich, St. Louis, MO, USA). RNA was extracted following treatment with isopropanol and sodium acetate ( $\mathrm{pH}$ 5.2). After washing with $75 \%$ ethanol, the RNA purity was measured using a NanoDrop 1000 ultraviolet spectrophotometer (Thermo Fisher Scientific, Waltham, MA, USA).

Reverse transcription-quantitative PCR. Total RNA $(1 \mu \mathrm{g})$ was reverse transcribed into cDNA in a $20-\mu 1$ reaction volume using a PrimeScript miRNA qPCR starter kit (version 2.0; Takara Biotechnology Co. Ltd., Dalian, China), according to the manufacturer's instructions. LET-7d, LET-7g and LET-7i were used as internal standards (17). Quantitative PCR was performed with a PCR 7500 (Applied Biosystems
Table I. Primers used for reverse transcription polymerase chain reaction.

\begin{tabular}{ll}
\hline miRNA & \multicolumn{1}{c}{ Primer } \\
\hline miR-93 & CAAAGTGCTGTTCGTGCAGGTAG \\
miR-320 & AAAAGCTGGGTTGAGAGGGCGA \\
miR-16 & TAGCAGCACGTAAATATTGGCG \\
LET-7d & AGAGGTAGTAGGTTGCATAGTT \\
LET-7g & TGAGGTAGTAGTTTGTACAGTT \\
LET-7i & TGAGGTAGTAGTTTGTGCTGTT
\end{tabular}

miR, microRNA.

Life Technologies, Foster City, CA, USA) at the following thermal cycling conditions: One cycle of $30 \mathrm{sec}$ at $95^{\circ} \mathrm{C}$, followed by 40 cycles of $5 \mathrm{sec}$ at $95^{\circ} \mathrm{C}$ and $34 \mathrm{sec}$ at $60^{\circ} \mathrm{C}$. The data were presented as the relative expression level of the gene of interest compared with the internal control gene, as determined using the $2^{-\Delta \Delta \mathrm{Ct}}$ method as follows: $\Delta \mathrm{Ct}=\mathrm{Ct}_{\text {miRnA }}-1 / 3\left(\mathrm{Ct}_{\text {LET-7d }}+\mathrm{Ct}_{\text {LET-7g }}+\mathrm{Ct}_{\text {LET-7i }}\right)$. Primers (miR-93, miR-320, miR-16, LEt-7d, LEt-7g and LEt-7i) were purchased from Invitrogen Life Technologies (Carlsbad, CA, USA) and the sequences are listed in Table I. Each experiment was repeated three times.

Statistical analysis. Statistical analysis was performed using SPSS software, version 17.0 (SPSS, Inc, Chicago, IL, USA). Data are presented as the mean \pm standard deviation. Comparisons between groups were performed using the t-test or the $\chi^{2}$ test. Pearson's correlation and linear regression analyses were used to determine the associations between certain indices in the different groups. In addition, receiver operating characteristic (ROC) curves were used to assess the sensitivity and specificity of miR-93, miR-320 and miR-16 as diagnostic markers for $\mathrm{ED}$. $\mathrm{P}<0.05$ was considered to indicate a statistically significant difference.

\section{Results}

Comparison of the clinical data. As shown in Table II, there were no statistically significant differences with regard to age in the three groups $(\mathrm{P}>0.05)$, and there was no statistically significant difference in the duration of diabetes or the BMI between the ED and NED groups $(\mathrm{P}>0.05)$. When compared with the control group, the BMI was evidently increased in the ED and NED groups $(\mathrm{P}<0.001$ and $\mathrm{P}<0.05$, respectively). The differences in FPG, TC and TG levels between the ED and NED groups were not statistically significant different ( $>0.05)$; however, the indices in the ED and NED groups were statistically different when compared with the control group $(\mathrm{P}<0.05)$. Furthermore, the TT level was much lower in the ED group when compared with the NED and control groups $(\mathrm{P}<0.05)$. In addition, the incidence rate of MA in the ED group was similar to that in the NED group (52.5 vs. 55\%, $\mathrm{P}>0.05)$; however, the incidence rate of DR (37.5\%) and DN $(40 \%)$ was higher in the ED group when compared with the NED group (10 and $17.5 \%$, respectively; $\mathrm{P}<0.05$ ). 
Table II. Comparisons of clinical data in three groups.

\begin{tabular}{|c|c|c|c|c|c|c|}
\hline Parameter & ED group & NED group & Control group & $\mathrm{P} 1$ & $\mathrm{P} 2$ & P3 \\
\hline Age, years & $47.37 \pm 5.81$ & $46.05 \pm 4.88$ & $46.9 \pm 5.69$ & 0.273 & 0.713 & 0.476 \\
\hline $\begin{array}{l}\text { Diabetes } \\
\text { duration, years }\end{array}$ & $5.40 \pm 2.56$ & $5.72 \pm 2.33$ & - & 0.554 & - & - \\
\hline BMI, $\mathrm{kg} / \mathrm{m}^{2}$ & $26.2 \pm 3.47$ & $25.90 \pm 3.49$ & $23.79 \pm 3.47$ & 0.721 & $<0.001$ & 0.002 \\
\hline IIEF-5 score & $16.97 \pm 3.95$ & $23.82 \pm 0.78$ & $23.80 \pm 0.78$ & $<0.001$ & $<0.001$ & 0.885 \\
\hline $\mathrm{FPG}, \mathrm{mmol} / \mathrm{l}$ & $10.79 \pm 3.22$ & $11.07 \pm 2.47$ & $4.23 \pm 1.08$ & 0.657 & $<0.001$ & $<0.001$ \\
\hline HbA1c, $\%$ & $8.22 \pm 1.08$ & $7.73 \pm 1.02$ & $4.97 \pm 0.83$ & 0.039 & $<0.001$ & $<0.001$ \\
\hline $\mathrm{TC}, \mathrm{mmol} / \mathrm{l}$ & $5.47 \pm 0.70$ & $5.27 \pm 0.66$ & $4.17 \pm 0.62$ & 0.181 & $<0.001$ & $<0.001$ \\
\hline $\mathrm{TG}, \mathrm{mmol} / \mathrm{l}$ & $1.64 \pm 1.31$ & $1.32 \pm 0.35$ & $1.07 \pm 0.42$ & 0.144 & 0.011 & 0.005 \\
\hline $\mathrm{TT}, \mathrm{ng} / \mathrm{ml}$ & $3.69 \pm 0.78$ & $4.29 \pm 1.11$ & $4.53 \pm 0.97$ & 0.006 & $<0.001$ & 0.305 \\
\hline MA, n (\%) & $21(52.5)$ & $22(55)$ & - & 0.823 & - & - \\
\hline DR, n (\%) & $15(37.5)$ & $4(10)$ & - & 0.004 & - & - \\
\hline DN, n (\%) & $16(40)$ & $7(17.5)$ & - & 0.026 & - & - \\
\hline
\end{tabular}

Data are presented as the mean \pm standard deviation ( $\mathrm{n}=40$ per group). P1, ED group vs. NED group; P2, ED group vs. control group; P3, NED group vs. control group. ED, erectile dysfunction; NED, diabetic without erectile dysfunction; BMI, body mass index; IIEF, international index of erectile function; FPG, fasting plasma glucose; HbA1c, glycated hemoglobin; TC, total cholesterol; TG, triglyceride; TT, testosterone; MA, macroangiopathy; DR, diabetic retinopathy; DN, diabetic nephropathy.

Table III. Correlation analysis between the incidence of diabetic erectile dysfunction and other indices.

\begin{tabular}{|c|c|c|c|c|c|c|}
\hline Correlation & BMI & FPG & $\mathrm{HbA} 1 \mathrm{c}$ & $\mathrm{TC}$ & $\mathrm{TG}$ & $\mathrm{TT}$ \\
\hline $\mathrm{R}$-value & 0.41 & -0.05 & 0.231 & 0.151 & 0.165 & -0.302 \\
\hline P-value & 0.721 & 0.657 & 0.039 & 0.181 & 0.144 & 0.006 \\
\hline
\end{tabular}

BMI, body mass index; FPG, fasting plasma glucose; HbA1c, glycated hemoglobin; TC, total cholesterol; TG, triglyceride; TT, testosterone.

Table IV. Correlations between erectile dysfunction and biochemical parameters using logistic regression analysis.

\begin{tabular}{lccccrr}
\hline Statistic & BMI & FPG & HbA1c & TC & TG & TT \\
\hline$\beta$-value & 0.04 & 0.066 & 0.578 & 0.298 & 0.351 & 0.828 \\
SE & 0.81 & 0.105 & 0.267 & 0.390 & 0.355 & 0.325 \\
P-value & 0.623 & 0.528 & 0.03 & 1.347 & 0.324 & 1.420 \\
OR & 1.040 & 1.069 & 1.783 & $0.627-2.895$ & $0.708-2.850$ & $0.231-0.827$ \\
$95 \%$ CI & $0.888-1.219$ & $0.869-1.314$ & $1.057-3.007$ & 0.037 \\
\hline
\end{tabular}

SE, standard error; OR, odds ratio; CI, confidence interval; BMI, body mass index; FPG, fasting plasma glucose; HbA1c, glycated hemoglobin; TC, total cholesterol; TG, triglyceride; TT, testosterone.

Relative factor and logistic regression analysis. Pearson's correlation analysis indicated that the incidence of diabetic ED negatively correlated with the serum TT levels $(r=0.302$, $\mathrm{P}<0.05$ ); however, a positive correlation was observed with the HbA1c levels ( $\mathrm{r}=0.231, \mathrm{P}<0.05$; Table III).

The predictor-dependent variable was represented by the presence of ED, while the independent variables were BMI, FPG, HbA1c, TC, TG and TT. Logistic regression analysis (Table IV) revealed that HbAlc and TT levels were impacting factors on diabetic $\mathrm{ED}(\mathrm{P}<0.05)$, and that the odds ratio values of HbAlc and TT were 1.783 [95\% confidence interval (CI), 1.057-3.007] and 0.437 (95\% CI, 0.231-0.827), respectively, which indicated that $\mathrm{HbAlc}$ was a risk factor and TT was a protective factor for diabetic ED. Specifically, a high HbAlc level or a low TT level promoted the incidence of diabetic ED.

Relative expression levels of miR-93, miR-320 and miR-16. Expression levels of miR-93, miR-320 and miR-16 were higher in the ED group when compared with the NED and control groups $(\mathrm{P}<0.05$; Table $\mathrm{V})$. 
Table V. Relative expression levels of miR-93, miR-320 and miR-16 in the three groups.

\begin{tabular}{lccccc}
\hline miRNA & ED group & NED group & Control group & P1 & P2 \\
\hline miR-93 $\left(2^{-\Delta \Delta C t}\right)$ & $0.98 \pm 0.31$ & $0.75 \pm 0.28$ & $0.66 \pm 0.19$ & $<0.05$ & $<0.05$ \\
miR-320 $\left(2^{-\Delta \Delta C t}\right)$ & $4.33 \pm 0.90$ & $3.19 \pm 0.86$ & $3.09 \pm 0.95$ & $<0.05$ & $<0.05$ \\
miR-16 $\left(2^{-\Delta \Delta \mathrm{Ct}}\right)$ & $1.06 \pm 0.30$ & $0.71 \pm 0.22$ & $0.69 \pm 0.28$ & $<0.05$ & $<0.05$ \\
\hline
\end{tabular}

Data are presented as the mean \pm standard deviation. P1, ED group vs. NED group; P2, ED group vs. control group; P3, NED group vs. control group. miR, microRNA; ED, erectile dysfunction; NED, diabetic without erectile dysfunction.
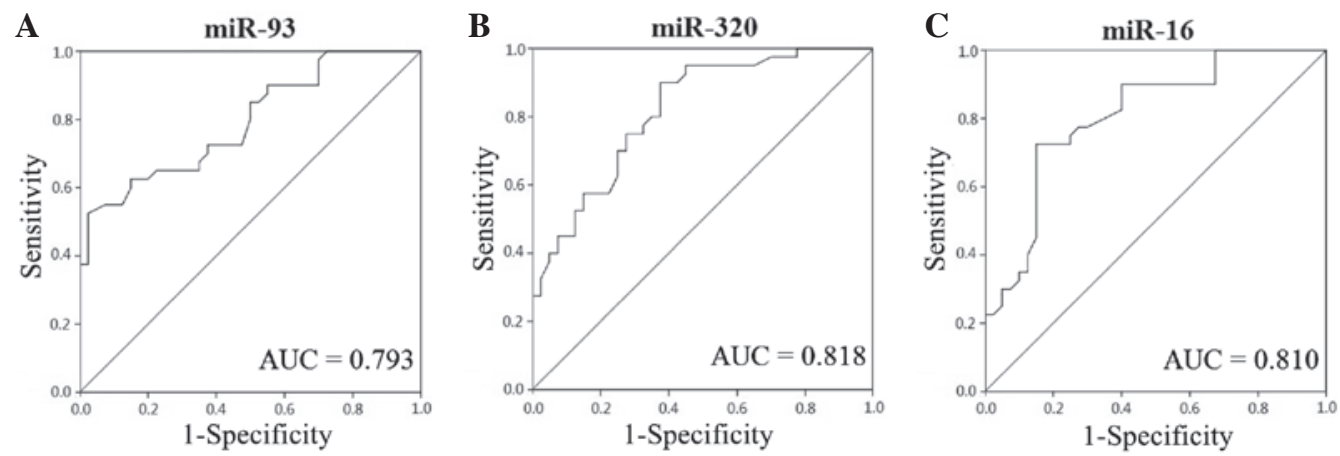

ED and control group
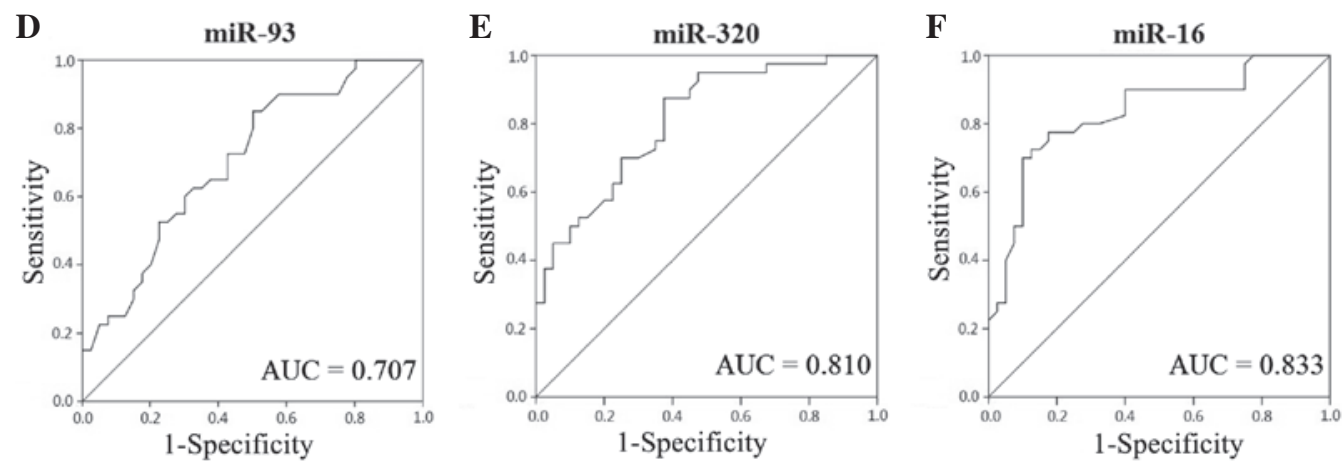

ED and NED group
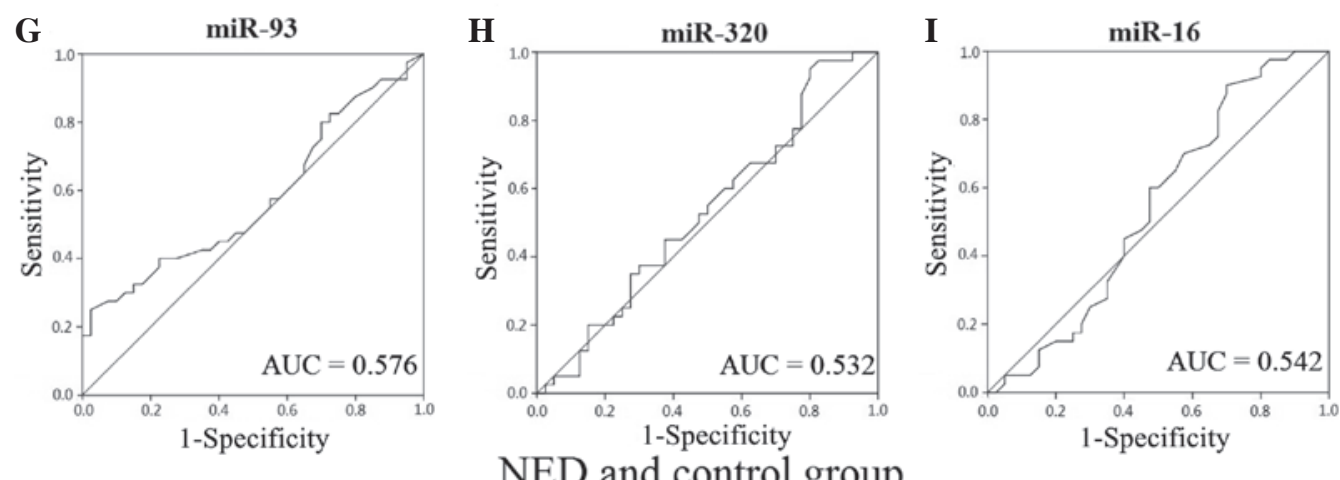

NED and control group

Figure 1. Receiver operating characteristic (ROC) curves and the calculated AUC values for miR-93, miR-320 and miR-16. Representative ROC curves for the three miRNAs when comparing the (A-C) ED and control groups, (D-F) ED and NED groups, and (G-I) NED and control groups. miR, microRNA; ED, erectile dysfunction; NED, diabetes without erectile dysfunction; AUC, area under the curve.

ROC curves for $m i R-93, m i R-320$ and $m i R-16$. When compared with the control group, the area under the curve (AUC) values for miR-93, miR-320 and miR-16 in the ED group were 0.793 (95\% CI, 0.696-0.890), 0.818 (95\% CI, 0.728-0.909) and
0.810 (95\% CI, 0.715-0.905), respectively. Additionally, when compared with the control group, the AUC values for miR-93, miR-320 and miR-16 in the NED group were $0.576(95 \% \mathrm{CI}$, 0.449-0.702), 0.532 (95\% CI, 0.405-0.660) and 0.542 (95\% CI, 
0.412-0.671), respectively. When compared with the NED group, the AUC values for miR-93, miR-320 and miR-16 in the ED group were 0.707 (95\% CI, 0.594-0.820), 0.810 (95\% CI, $0.718-0.903)$ and 0.833 (95\% CI, 0.743-0.924), respectively (Fig. 1). The AUC results indicated that miR-93, miR-320 and miR-16 had a diagnostic value in diabetic ED.

\section{Discussion}

Diabetes comprises a number of metabolic diseases in which the blood sugar levels are high over a prolonged period $(18,19)$. With a population increasing in age, a reduction in sporting activity and a high incidence of obesity, the number of individuals suffering from diabetes is increasing. ED is characterized by the regular or repeated inability to maintain an erection and is one of the most common complications of diabetes. Although ED is a benign disease, the condition impacts the physical and mental health of the patient, and is closely associated with the quality of life, the relationship with a sexual partner and family stability. A retrospective study that analyzed diabetic complications in the USA over the past 20 years found that since the prevalence of diabetes continues to increase, diabetes-associated complications have become a huge burden of the disease (20).

The risk factors of diabetic ED include high HbAlc levels, a high BMI, insulin resistance and angiopathy $(4,21,22)$. Diabetic macroangiopathy, microangiopathy and endothelial dysfunction are all involved in the pathophysiological progression of ED. Briefly, diabetic microangiopathy causes atherosclerosis and thrombosis, which leads to the narrowing of the artery lumen, subsequently reducing bloodflow to the penis. In addition, microangiopathy is involved in ED through affecting autonomic neuropathies (23-27). In the present study, the results demonstrated that the incidence of DR and DN was significantly higher in the ED group compared with the NED group $(\mathrm{P}<0.05)$, which indicated that diabetic patients with ED suffered from severe microangiopathy. Hermans et al (28) reported similar results.

Changes in the androgenic level are also an important factor in diabetic ED $(29,30)$. Diabetes affects the secretion of androgen, which plays a key role in maintaining sexual desire; therefore, low androgenic levels caused by diabetes reduces sexual desire, which leads to the incidence of ED. The results of the present study revealed that the TT level was significantly lower in the ED group when compared with the NED group $(\mathrm{P}<0.05)$. According to the logistic regression analysis, the TT level was a protective factor in diabetic ED; thus, the incidence of diabetic ED may increase when the TT level is lower than the normal level. Kataoka et al (31) found that androgen replacement therapy for erectile function in rats with type 2 diabetes was able to increase the intracranial/mean arterial pressure ratio to suppress inflammation and metabolic disorders and improve the endothelial and erectile functions. By contrast, the $\mathrm{HbA1c}$ level is a risk factor in diabetic ED; a high $\mathrm{HbA1c}$ level promotes the incidence of diabetic ED (32). In addition, the HbAlc level is proportional to the average blood glucose concentration over the previous four weeks to three months. The long-term dyscontrol of blood sugar levels means that the chronic complications of patients with diabetes are likely to deteriorate, including diabetic angiopathy, which may aggra- vate the conditions of diabetic ED. In addition, a high $\mathrm{HbAlc}$ level is known to indicate long-term high blood glucose levels, which affect the function of the hypothalamic-pituitary-gonad axis, causing the reduction of gonadotropin and follicle-stimulating hormone secretion (33). Subsequently, changes are inflicted on Leydig cells, which ultimately impacts penile erectile function (34).

miRNA are small non-coding RNA molecules of $\sim 22$ nucleotides that are found in animals and plants. The molecules function in transcription and post-transcription to negatively regulate gene expression. miRNA play a critical role in the incidence of diabetes and diabetic complications (35). Wang et al (36) reported that the expression of miR-320 was increased in myocardial microvascular endothelial cells (MMVECs), and that the target genes may be insulin-like growth factor (IGF)-1 and IGF-1 receptor. It was found that the upregulation of miR-320 in MMVECs from Goto-Kakizaki rats may be responsible for the inconsistency between the expression of IGF-1 protein and mRNA; therefore, miR-320 may be associated with impaired angiogenesis in diabetes (36). Furthermore, Long et al (37) demonstrated that transfection of miR-93 prevented the effect of high glucose on vascular endothelial growth factor (VEGF) downstream targets; thus, tropic VEGF levels were increased through inhibition of miR-93 gene expression. Villeneuve et al $(38,39)$ found that miR-125b may increase the expression of inflammatory genes in vascular endothelial cells to cause endothelial dysfunction. Weber et al (40) and Fleissner et al (41) also found that miR-21 was able to reduce the rate of apoptosis in vascular endothelial cells and promote the release of nitric oxide to protect the vascular endothelium. Caporali et al (42) reported that inhibition of miR-15 improved endothelial function in culture conditions mimicking diabetes mellitus (high D-glucose), and CCNE1 and CDC25A were direct miR-15 targets. The present study found that miR-93, miR-320 and miR-16 were highly expressed in the diabetic ED group, indicating that diabetic angiopathy played an important role in the onset of ED in patients with diabetes.

In conclusion, the results of the current study demonstrated that the HbA1c level was a risk factor for diabetic ED, while the TT level was a protective factor, which was the same as a previous result; however, the BMI, FPG, TC and TG did not show a correlation with diabetic ED (4,21,22,29-31). These results may have been caused by the small sample size. In addition, detecting the levels of miR-93, miR-320 and miR-16 through a quantitative PCR assay and ROC curve analysis may aid the diagnosis of diabetic ED. However, whether additional miRNAs exhibit correlations with diabetic ED was unable to be determined due to the small sample size and certain restrictions. Therefore, the present study confirmed that detecting the levels of miR-93, miR-320 and miR-16 may be significant for the diagnosis of diabetic ED. Due to the complex mechanisms of diabetic ED and the numerous factors involved, the exact mechanisms underlying diabetic ED require further study in the future.

\section{References}

1. Yang W, Lu J, Weng J, et al: Prevalence of diabetes among men and women in China. N Engl J Med 362: 1090-1101, 2010. 
2. Montorsi F, Adaikan G, Becher E, et al: Summary of the recommendations on sexual dysfunctions in men. J Sex Med 7: 3572-3588, 2010

3. Ma RC, So WY, Yang X, et al: Erectile dysfunction predicts coronary heart disease in type 2 diabetes. J Am Coll Cardiol 51: 2045-2050, 2008.

4. Cho NH, Ahn CW, Park JY, et al: Prevalence of erectile dysfunction in Korean men with Type 2 diabetes mellitus. Diabet Med 23: 198-203, 2006

5. Chew SK, Taouk Y, Xie J, et al: The relationship of retinal vessel caliber with erectile dysfunction in patients with type 2 diabetes. Invest Ophthalmol Vis Sci 54: 7234-7239, 2013.

6. Canat L, Cicek G, Atis G, Gurbuz C and Caskurlu T: Is there a relationship between severity of coronary artery disease and severity of erectile dysfunction? Int Braz J Urol 39: 465-473, 2013.

7. Lin F and Gou X: Panax notoginseng saponins improve the erectile dysfunction in diabetic rats by protecting the endothelial function of the penile corpus cavernosum. Int J Impot Res 25 : 206-211, 2013.

8. Rosen RC, Riley A, Wagner G, Osterloh IH, Kirkpatrick J and Mishra A: The international index of erectile function (IIEF) A multidimensional scale for assessment of erectile dysfunction. Urology 49: 822-830, 1997.

9. Hatzichristou D, Hatzimouratidis K, Bekas M, Apostolidis A, Tzortzis V and Yannakoyorgos K: Diagnostic steps in the evaluation of patients with erectile dysfunction. J Urol 168: 615-620, 2002.

10. Hatzimouratidis K, Amar E, Eardley I, et al; European Association of Urology: Guidelines on male sexual dysfunction: Erectile dysfunction and premature ejaculation. Eur Urol 57: 804-814, 2010

11. Kloosterman WP and Plasterk RH: The diverse functions of microRNAs in animal development and disease. Dev Cell 11: 441-450, 2006

12. Heneghan HM, Miller N, McAnena OJ, O'Brien T and Kerin MJ: Differential miRNA expression in omental adipose tissue and in the circulation of obese patients identifies novel metabolic biomarkers. J Clin Endocrinol Metab 96: E846-E850, 2011

13. Trajkovski M, Hausser J, Soutschek J, et al: MicroRNAs 103 and 107 regulate insulin sensitivity. Nature 474: 649-653, 2011.

14. Zampetaki A, Kiechl S, Drozdov I, et al: Plasma microRNA profiling reveals loss of endothelial miR-126 and other microRNAs in type 2 diabetes. Circ Res 107: 810-817, 2010.

15. Kong L, Zhu J, Han W, et al: Significance of serum microRNAs in pre-diabetes and newly diagnosed type 2 diabetes: A clinical study. Acta Diabetol 48: 61-69, 2011

16. World Health Organization: Definition, diagnosis and classification of diabetes mellitus and its complications. Report of a WHO consultation. Part 1: diagnosis and classification of diabetes mellitus. Geneva: World Health Organization, 1999.

17. Chen X, Liang H, Guan D, et al: A combination of Let-7d, Let-7g and Let-7i serves as a stable reference for normalization of serum microRNAs. PLoS One 8: e79652, 2013.

18. McKinlay J and Marceau L: US public health and the $21 \mathrm{st}$ century: Diabetes mellitus. Lancet 356: 757-761, 2000.

19. Tricco AC, Ivers NM, Grimshaw JM, et al: Effectiveness of quality improvement strategies on the management of diabetes: A systematic review and meta-analysis. Lancet 379: 2252-2261, 2012.

20. Gregg EW, Li Y, Wang J, et al: Changes in diabetes-related complications in the United States, 1990-2010. N Engl J Med 370 1514-1523, 2014

21. Ahmed I, Aamir Au, Anwar E, Ali SS, Ali A and Ali A: Erectile dysfunction and type 2 diabetes mellitus in northern Pakistan. J Pak Med Assoc 63: 1486-1490, 2013.

22. Yang G, Pan C and Lu J: Prevalence of erectile dysfunction among Chinese men with type 2 diabetes mellitus. Int J Impot Res 22: 310-317, 2010.

23. Malavige LS and Levy JC: Erectile dysfunction in diabetes mellitus. J Sex Med 6: 1232-1247, 2009.
24. Ponholzer A, Stopfer J, Bayer G, et al: Is penile atherosclerosis the link between erectile dysfunction and cardiovascular risk? An autopsy study. Int J Impot Res 24: 137-140, 2012.

25. Ellati RT, Dokun AO, Kavoussi PK, Steers WD, Annex BH and Lysiak JJ: Increased phosphodiesterase type 5 levels in a mouse model of type 2 diabetes mellitus. J Sex Med 10: 362-369, 2013.

26. Angulo J, González-Corrochano R, Cuevas P, et al: Diabetes exacerbates the functional deficiency of NO/cGMP pathway associated with erectile dysfunction in human corpus cavernosum and penile arteries. J Sex Med 7: 758-768, 2010.

27. Qiu XF, Li XX, Chen Y, et al: Mobilisation of endothelial progenitor cells: One of the possible mechanisms involved in the chronic administration of melatonin preventing erectile dysfunction in diabetic rats. Asian J Androl 14: 481-486, 2012.

28. Hermans MP, Ahn SA and Rousseau MF: Erectile dysfunction, microangiopathy and UKPDS risk in type 2 diabetes. Diabetes Metab 35: 484-489, 2009.

29. Corona G and Maggi M: Patients with testosterone deficiency syndrome and type 2 diabetes. Arch Esp Urol 66: 711-722, 2013.

30. Hackett G, Cole N, Bhartia M, Kennedy D, Raju J and Wilkinson P: Testosterone replacement therapy with long-acting testosterone undecanoate improves sexual function and quality-of-life parameters vs. placebo in a population of men with type 2 diabetes. J Sex Med 10: 1612-1627, 2013.

31. Kataoka T, Hotta Y, Maeda Y and Kimura K: Assessment of androgen replacement therapy for erectile function in rats with type 2 diabetes mellitus by examining nitric oxide-related and inflammatory factors. J Sex Med 11: 920-929, 2014.

32. Cheng D, Fei Y, Liu Y, Li J, Xue Q, Wang X and Wang N: HbA1C variability and the risk of renal status progression in Diabetes Mellitus: A meta-analysis. PLoS One 9: e115509, 2014.

33. Juarez DT, Demaris KM, Goo R, Mnatzaganian CL and Wong Smith H: Significance of $\mathrm{HbAlc}$ and its measurement in the diagnosis of diabetes mellitus: US experience. Diabetes Metab Syndr Obes 7: 487-494, 2014

34. Sabanayagam C, Khoo EY, Lye WK, et al: Diagnosis of diabetes mellitus using HbAlc in Asians: Relationship between HbAlc and retinopathy in a multiethnic Asian population. J Clin Endocrinol Metab 100: 689-696, 2015.

35. Natarajan R, Putta S and Kato M: MicroRNAs and diabetic complications. J Cardiovasc Transl Res 5: 413-422, 2012.

36. Wang XH, Qian RZ, Zhang W, Chen SF, Jin HM and Hu RM: MicroRNA-320 expression in myocardial microvascular endothelial cells and its relationship with insulin-like growth factor-1 in type 2 diabetic rats. Clin Exp Pharmacol Physiol 36: 181-188, 2009.

37. Long J, Wang Y, Wang W, Chang BH and Danesh FR: Identification of microRNA-93 as a novel regulator of vascular endothelial growth factor in hyperglycemic conditions. J Biol Chem 285: 23457-23465, 2010 .

38. Villeneuve LM, Reddy MA, Lanting LL, Wang M, Meng L and Natarajan R: Epigenetic histone H3 lysine 9 methylation in metabolic memory and inflammatory phenotype of vascular smooth muscle cells in diabetes. Proc Natl Acad Sci USA 105 9047-9052, 2008.

39. Villeneuve LM, Kato M, Reddy MA, Wang M, Lanting L and Natarajan R: Enhanced levels of microRNA-125b in vascular smooth muscle cells of diabetic $\mathrm{db} / \mathrm{db}$ mice lead to increased inflammatory gene expression by targeting the histone methyltransferase Suv39h1. Diabetes 59: 2904-2915, 2010.

40. Weber M, Baker MB, Moore JP and Searles CD: MiR-21 is induced in endothelial cells by shear stress and modulates apoptosis and eNOS activity. Biochem Biophys Res Commun 393: 643-648, 2010.

41. Fleissner F, Jazbutyte V, Fiedler J, et al: Short communication: Asymmetric dimethylarginine impairs angiogenic progenitor cell function in patients with coronary artery disease through a microRNA-21-dependent mechanism. Circ Res 107: 138-143, 2010

42. Caporali A, Meloni M, Völlenkle C et al: Deregulation of microRNA-503 contributes to diabetes mellitus-induced impairment of endothelial function and reparative angiogenesis after limb ischemia. Circulation 123: 282-291, 2011. 